\title{
Parking Lot Sealcoat: A Major Source of Polycyclic Aromatic Hydrocarbons (PAHs) in Urban and Suburban Environments
}

\author{
By Peter C. Van Metre', Barbara J. Mahler', Mateo Scoggins², and Pixie A. Hamilton ${ }^{1}$
}

Collaborative studies by the City of Austin and the U. S. Geological Survey (USGS) have identified coal-tar based sealcoat - the black, shiny emulsion painted or sprayed on asphalt pavement such as parking lots-as a major and previously unrecognized source of polycyclic aromatic hydrocarbon (PAH) contamination. Several PAHs are suspected human carcinogens and are toxic to aquatic life. Studies in Austin, Texas, showed that particles in runoff from coal-tar based sealcoated parking lots had concentrations of PAHs that were about 65 times higher than concentrations in particles washed off parking lots that had not been sealcoated. Biological studies, conducted by the City of Austin in the field and in the laboratory, indicated that PAH levels in sediment contaminated with abraded sealcoat were toxic to aquatic life and were degrading aquatic communities, as indicated by loss of species and decreased numbers of organisms. Identification of this source of PAHs may help to improve future strategies for controlling these compounds in urban water bodies across the Nation where parking lot sealcoat is used.

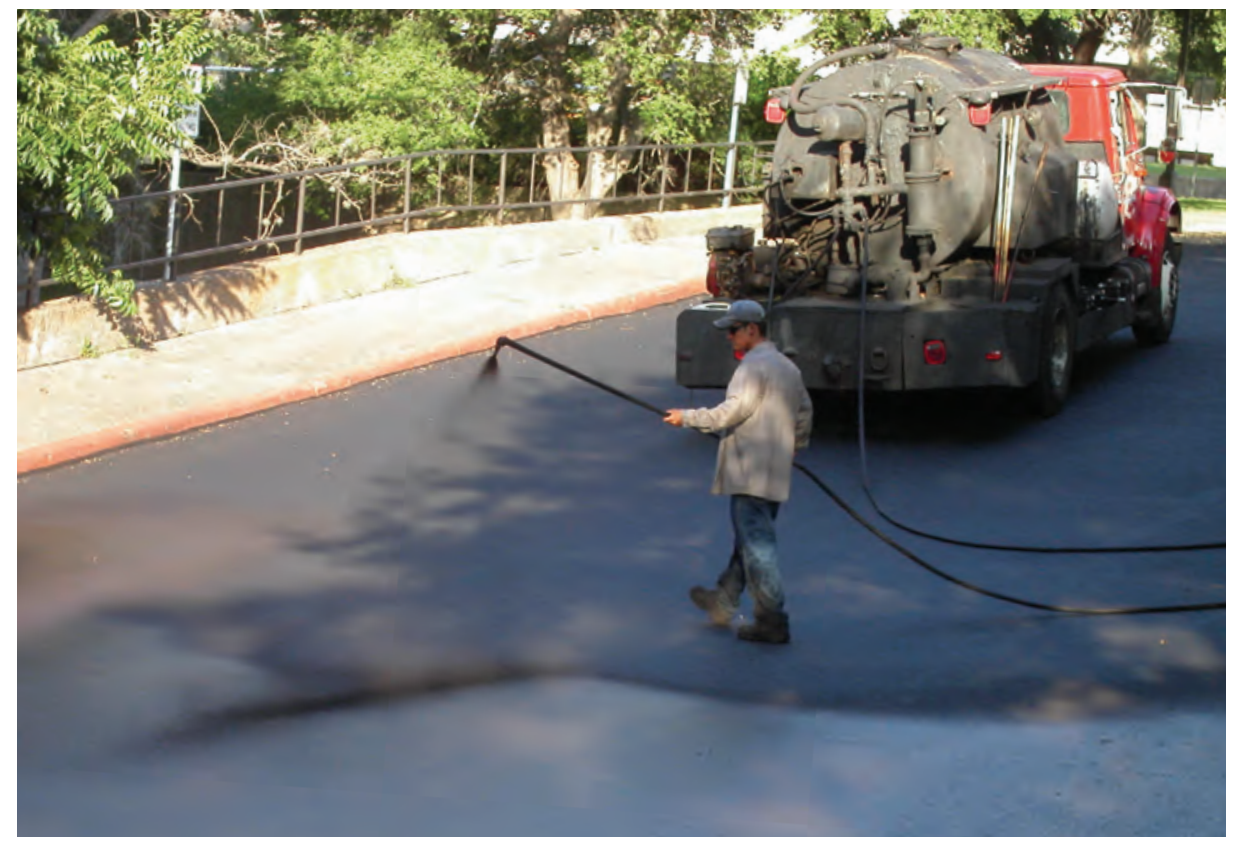

Particles in runoff from coal-tar based sealcoated parking lots had concentrations of PAHs that are about 65 times higher than concentrations in particles washed off parking lots that had not been sealcoated.

\section{What are PAHs, coal tar, and sealcoat?}

Polycyclic aromatic hydrocarbons (or PAHs) are a group of organic contaminants that form from the incomplete combustion of hydrocarbons, such as coal and gasoline. PAHs are an environmental concern because they are toxic to aquatic life and because several are suspected human carcinogens.

Coal tar is a byproduct of the coking of coal, and can contain 50 percent or more PAHs by weight.

Sealcoat is a black liquid that is sprayed or painted on asphalt pavement in an effort to protect and beautify the asphalt. Most sealcoat products are coal-tar or asphalt based. Many coal-tar sealcoat products contain as much as 30 percent coal tar by weight. Product analyses by the City of Austin indicated that coal-tar sealcoat products have median concentrations of total PAHs about 70 times higher than concentrations in asphalt-based sealcoat products.

Sealcoat is used commercially and by homeowners across the Nation. It is applied to residential driveways and to parking lots associated with commercial businesses (including strip malls and shopping centers), apartment and condominium complexes, churches, schools, and business parks. The City of Austin, Texas, estimates that about 600,000 gallons of sealcoat are applied every year in the greater Austin area. National use numbers are not available, but commercial availability suggests that asphaltbased sealcoat is commonly used on the West Coast and coal-tar based sealcoat is commonly used in the Midwest, the South, and on the East Coast. 


\section{How did USGS study parking lot runoff?}

USGS researchers sampled runoff at 13 parking lots in Austin. They also took scraping samples of parking lot surfaces to compare source materials to particles in the runoff. Scraping samples and the water and particles in the runoff samples were analyzed for a suite of PAHs, major elements, and trace elements. The researchers sprayed water on four different types of parking lot surfaces: lots sealed with coal-tar based sealcoat (top photo), lots sealed with asphalt-based sealcoat, unsealed asphalt lots, and unsealed concrete lots. The runoff was collected behind spill berms, pumped into containers (middle photo) and filtered through Teflon filters to collect the particulates for analysis (bottom photo). The particulates, the filtered water, and samples of sealcoat scraped from the parking lot surfaces were analyzed for PAHs at the USGS National Water Quality Laboratory in Denver, Colorado. Concentrations and yields (the amount of PAHs coming off a parking lot per unit area) were used to determine levels of contamination in runoff from each type of parking lot and the importance of sealed lots as a source of PAHs to urban streams.

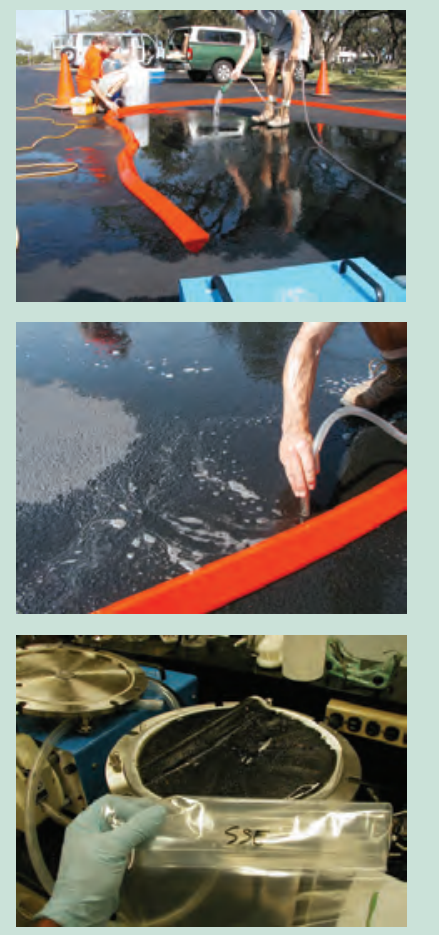

\section{How does sealcoat get from parking lots into the environment?}

Vehicle tires abrade parking lot sealcoat into small particles. These small particles are washed off parking lots by precipitation and into storm sewers and streams. Sealcoat "wear and tear" is visible in high traffic areas within a few months after application. Sealcoat manufacturers recommend reapplication every 2 to 3 years.

\section{What are potential environmental and human-health concerns?}

PAHs are toxic to mammals (including humans), birds, fish, amphibians, invertebrates, and plants. Aquatic invertebrates, the insects and other small animals that live in streams and lakes, are particularly susceptible to PAH contamination, especially the bottom dwellers (benthic invertebrates) that live in the mud where PAHs tend to accumulate. They are an important part of the food chain and are often monitored as indica- tors of stream quality (analogous to the "canary in the coal mine" concept). Possible effects of PAHs on aquatic invertebrates include inhibited reproduction, delayed emergence, sediment avoidance, and mortality, and possible adverse effects on fish include fin erosion, liver abnormalities, cataracts, and immune system impairments. PAHs tend to attach to sediment; the Probable Effect Concentration (PEC) - a widely used sedimentquality guideline that is the concentration of a contaminant in bed sediment expected to adversely affect benthic (or bottom-dwelling) biota—is $22.8 \mathrm{mg} / \mathrm{kg}$ (milligrams per kilogram) for total PAHs.

Studies by USGS and City of Austin did not evaluate human-health risk from exposure to sealcoat. Human-health risk from environmental contaminants is often evaluated in terms of exposure pathways. For example, people could potentially be exposed to PAHs in sealcoat through skin contact with abraded particles from parking lots, inhalation of wind-blown particles, and inhalation of fumes that in streams and lakes rarely pose a humanvolatilize from sealed parking lots. PAHs health risk via drinking water because of their tendency to attach to sediment rather than dissolve in water. In addition, because PAHs do not readily bioaccumulate within the food chain, possible human-health risks associated with consumption of fish are low.

\section{What are the concentrations of PAHs in runoff from sealed and unsealed parking lots?}

Concentrations of PAHs in particles (including abraded sealcoat along with urban dust and other sediment) washed off from each of the different surface types-including the unsealed parking lots-were greater than the PEC of $22.8 \mathrm{mg} / \mathrm{kg}$. The average concentration in particles washed off parking lots that were not sealcoated was $54 \mathrm{mg} / \mathrm{kg}$. This is not surprising because runoff from parking lots is likely to contain PAHs from many sources, including leaking motor oil, tire particles, vehicle exhaust, and atmospheric deposition.

Concentrations of PAHs were much higher in particles in runoff from parking lots sealed with coal-tar based sealcoat than from all other types of parking lot surfaces. Specifically, the average concentration of PAHs from coal-tar sealed lots was $3,500 \mathrm{mg} / \mathrm{kg}$, about 65 times higher than the average concentration in particles washed off unsealed parking lots. The average concentration in par-

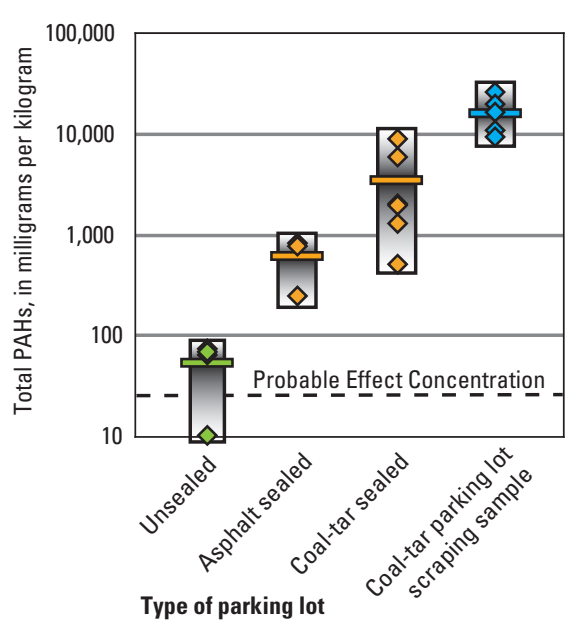

Concentrations of total PAHs in particles in runoff from sealed parking lots greatly exceeded concentrations from unsealed parking lots. The bar on each graph is the mean concentration. The $y$-axis is logarithmic. 
ticles washed off parking lots sealed with asphalt-based sealcoat was $620 \mathrm{mg} / \mathrm{kg}$, about 10 times higher than the average concentration from unsealed parking lots and 6 times less than the average concentration from coal-tar sealed lots.

The large differences between concentrations associated with sealed and unsealed parking lots indicate that abraded sealcoat is a major and previously unrecognized contributor to $\mathrm{PAH}$ contamination.

\section{How do PAHs from sealcoat affect the quality and biology of streams?}

Studies by USGS scientists demonstrated possible connections between PAHs in particles washed off sealed parking lots and PAHs in suspended sediment in four streams in Austin and Fort Worth, Texas. The total mass of PAHs (or "load") expected to wash off sealed parking lots was compared to the load of PAHs measured in suspended sediment in the four streams after rainstorms. The load of PAHs estimated to come from the sealed parking lots was comparable to the measured load in the streams, indicating that runoff from sealed parking lots could account for the majority of PAHs in these streams. Findings also showed that PAHs in suspended sediment in the streams were chemically similar to those in particles washed off parking lots sealed with coal-tar based sealcoat. What would be the effect on PAH loading to the streams if parking lots were not sealed? Estimates

\section{How did City of Austin scientists conduct biological studies?}

City of Austin biologists conducted laboratory and field studies to evaluate the effects of sealcoated parking lots on aquatic communities in area streams. These studies included toxicity testing in controlled laboratory experiments that exposed organisms to sediment spiked with coal-tar and asphalt-based sealcoat (left photo); controlled experiments that used aquariums with diverse natural biological communities to which sealcoat was added (middle photo); and field assessments of aquatic communities in streams upstream and downstream from inflows of runoff from sealcoated parking lots (right photo).
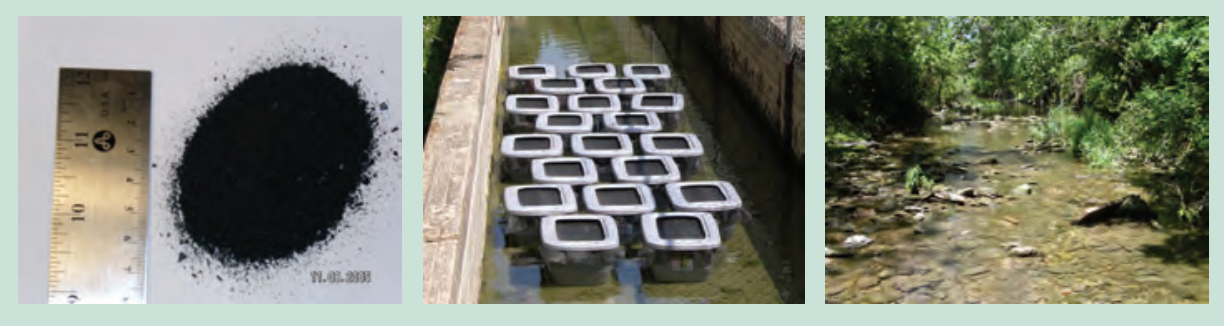

from the USGS study indicate that total loads of PAHs coming from parking lots in the studied watersheds would be reduced to about one-tenth of their current levels if all of the parking lots were unsealed.

Studies by City of Austin biologists showed that PAHs in sediment contaminated with abraded sealcoat could be adversely affecting aquatic communities. Specifically, toxicity testing of organisms in the laboratory showed large increases in mortality as sealcoat amounts and PAH concentrations were increased, and that sediment contaminated with coal-tar sealcoat was toxic to aquatic life at PAH concentrations observed in Austin water- ways. Controlled experiments that used aquariums with diverse natural biological communities showed significant biological degradation in response to the addition of sealcoat particles. Finally, field assessments in selected Austin streams showed loss of species and decreases in the number of aquatic organisms downstream from inflows of runoff from coaltar sealcoated parking lots. These effects coincided with increases in concentrations of PAHs in stream sediment below sealcoated parking lots. Overall, City of Austin scientists have reported PAH contamination at levels predicted to be toxic to benthic invertebrates in about 13 percent of sampled Austin creeks.
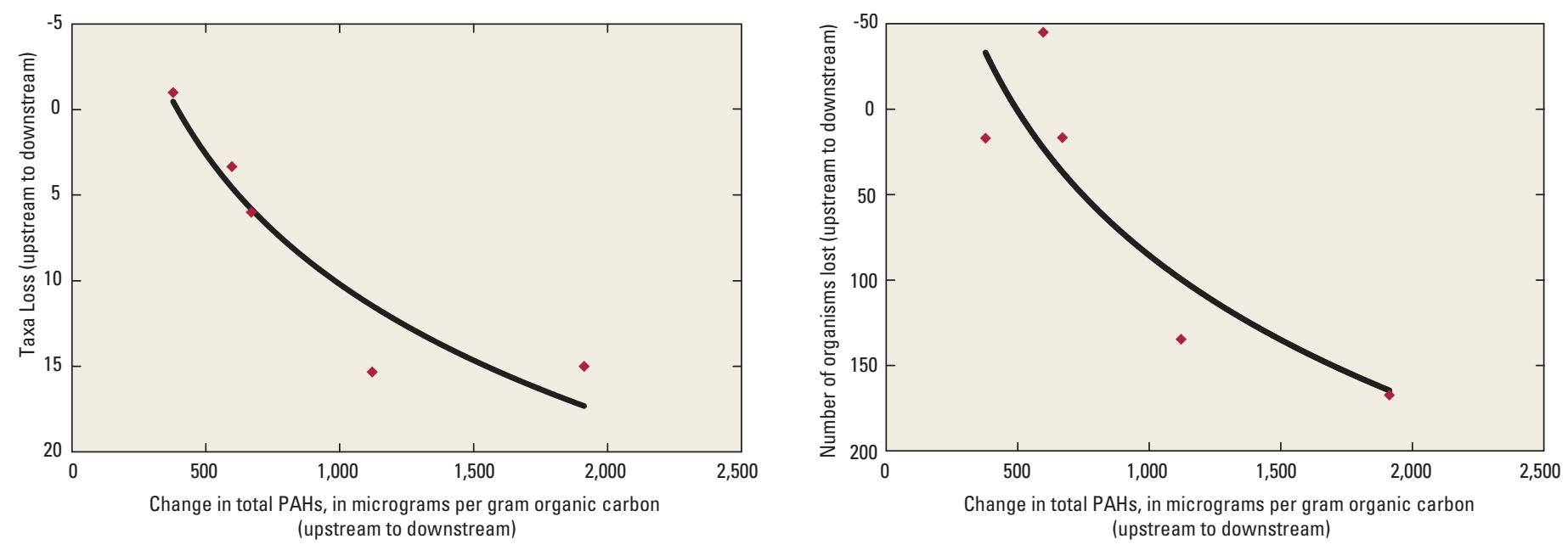

Field assessments in five selected Austin streams showed loss of species (taxa) and decreases in the number of aquatic organisms downstream of coal-tar sealcoated parking lots that can be, in large part, explained by increases in total PAHs. 


\section{How do these findings apply to urban lakes and reservoirs?}

The concentrations of PAHs in lakes and reservoirs across the Nation are increasing, as indicated by USGS studies of 38 reservoirs and lakes conducted in 18 metropolitan areas across the country (Van Metre and Mahler, 2005). Sediment cores (vertical tubes of mud) were collected from reservoir and lake bottoms (see photo below); analysis of these cores provides a reconstruction of historical water quality over time, much like using tree rings to reconstruct historical climate. Runoff carries soil, debris, and attached contaminants to lakes and reservoirs, which settle to the bottom; as the sediment builds up, changes in water quality are recorded in the successive sediment layers.

USGS findings show that concentrations of total PAHs in the majority of lakes and reservoirs in urban and suburban areas across the Nation increased sig-

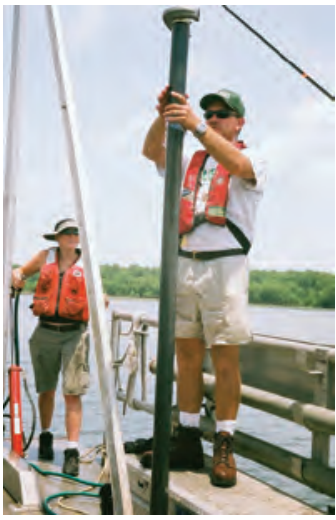
nificantly from 1970 to 2001. The increases were greatest in lakes with rapidly urbanizing watersheds (urban sprawl); for example, over the last 10 years, the concentrations of PAHs in

Lake in the Hills (suburban Chicago, Illinois) increased ten-fold as the watershed was rapidly developed. Further study is needed to assess direct links between the use of sealcoat and PAH trends in these urban lakes and reservoirs across the Nation.

\section{What are the implications of these studies?}

The study of parking lot surfaces by the USGS and the City of Austin show that abraded sealcoat could be a major source of PAHs to urban and suburban water bodies in watersheds across the Nation where sealcoat is used. Such findings have implications that extend beyond Texas as sealcoat is used nationwide; further studies would help to evaluate the potential impacts of sealcoat on the aquatic environment in other parts of the country. Identification of this source may influence future strategies for controlling PAHs in urban environments. In the past, sources of PAHs in urban watersheds were thought to be dominated by numerous nonpoint sources, such as leaking motor oil, tire wear, vehicular exhaust, and atmospheric deposition. Such sources are difficult to quantify or control because of their diffuse nature. In contrast, sealcoated parking lots are specific areas that contribute directly to urban stormwater runoff (see photo below), and the use of sealcoat is voluntary and controllable. To address PAH contamination in streams, the City of Austin Council banned the use of coal-tar based sealcoat, effective January 2006 (Nancy McClintock, written communication, City of Austin, November 2005).

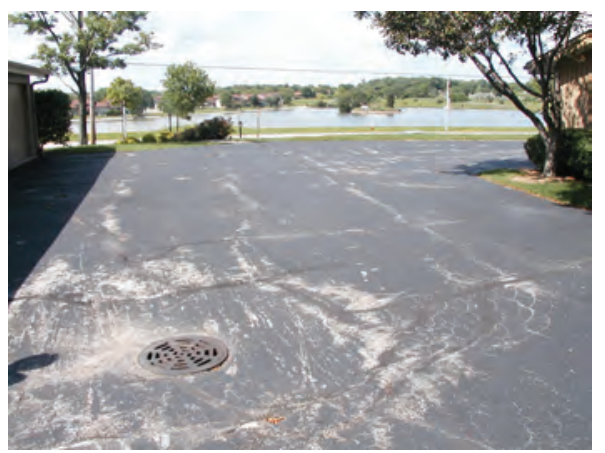

Possible alternatives to coal-tar based sealcoating of parking lots and driveways include the use of concrete and unsealed asphalt pavement, and the use of asphalt-based sealcoat that contains lower levels of PAHs.

Currently, the use of coal-tar based sealcoat is not federally regulated. In 1992, the U.S. Environmental Protection Agency excluded coke product residues, including coal tar, from classification as hazardous wastes if they are recycled. Under the Resource Conservation and Recovery Act, coal-tar based pavement sealants are products that contain recycled coal tar and, therefore, are not regulated.

\section{Contacts for additional information}

Peter Van Metre and Barbara Mahler

U.S. Geological Survey

8027 Exchange Drive

Austin, Texas 78754-4733

(512) 927-3506 or pcvanmet@usgs.gov

(512) 927-3566 or bjmahler@usgs.gov

\section{Mateo Scoggins}

City of Austin

Watershed Protection and Development

Review Department

505 Barton Springs Road, $11^{\text {th }}$ Floor

Austin, Texas 78704

(512) $974-1917$ or

mateo.scoggins@ci.austin.tx.us

\section{Links to related publications, data and maps}

\section{City of Austin Coal Tar Sealant Information- \\ http://www.ci.austin.tx.us/watershed/ bs_coaltar.htm}

USGS frequently asked questionshttp://water.usgs.gov/nawqa/asphalt_ sealers.html

Basic information on the toxicity of PAHs to biological organisms, U.S. Environmental Protection Agency (USEPA)http://www.epa.gov/R5Super/ecology/ html/toxprofiles.htm\#pahs

General information on PAH exposure, Agency for Toxic Substances and Disease Registry (ATSDR)http://www.atsdr.cdc.gov/toxprofiles/ phs69.html

\section{References}

Mahler, B.J., Van Metre, P.C., Bashara, T.J., Wilson, J.T., and Johns, D.A., 2005. Parking lot sealcoat: An unrecognized source of urban PAHs: Environmental Science and Technology, vol. 39 , no. 15, p. 5560-5566.

Van Metre, P.C. and Mahler, B.J., 2005. Trends in hydrophobic organic contaminants in urban and reference lake sediments across the United States, 1970-2001: Environmental Science and Technology, vol. 39, no. 15, p. 5567-5574.

City of Austin. 2005. PAHs in Austin, Texas. (http://www.ci.austin.tx.us/ watershed/downloads/coaltar_draft_ pah_study.pdf)

\footnotetext{
${ }^{1}$ U.S. Geological Survey
}

${ }^{2}$ City of Austin 